\title{
Preoperative prediction of gastrointestinal stromal tumors with high Ki-67 proliferation index based on CT features
}

\author{
Cai-Wei Yang ${ }^{1,2 \#} \wedge$, Xi-Jiao Liu ${ }^{2 \#} \wedge$, Lian Zhao ${ }^{2} \wedge$, Feng Che ${ }^{1} \wedge$, Yuan Yin $^{3} \wedge$, Hui-Jiao Chen ${ }^{4} \wedge$, Bo Zhang ${ }^{3} \wedge$, \\ Min $\mathrm{Wu}^{5,6,7} \wedge$, Bin Song ${ }^{2 \wedge}$ \\ ${ }^{1}$ West China School of Medicine, Sichuan University, Chengdu, China; ${ }^{2}$ Department of Radiology, West China Hospital, Sichuan University, \\ Chengdu, China; ${ }^{3}$ Department of Gastrointestinal Surgery, West China Hospital, Sichuan University, Chengdu, China; ${ }^{4}$ Department of Pathology, \\ West China Hospital, Sichuan University, Chengdu, China; ${ }^{5}$ Huaxi MR Research Center (HMRRC), Department of Radiology, West China \\ Hospital, Sichuan University, Chengdu, China; ${ }^{6}$ Department of Clinic Medical Center, Dazhou Central Hospital, Dazhou, China; ${ }^{7}$ Department of \\ Radiology, Molecular Imaging Program at Stanford (MIPS), Stanford University, Stanford, CA, USA \\ Contributions: (I) Conception and design: CW Yang, XJ Liu, B Song; (II) Administrative support: M Wu, B Song, B Zhang; (III) Provision of study \\ materials or patients: Y Yin, HJ Chen; (IV) Collection and assembly of data: CW Yang, F Che; (V) Data analysis and interpretation: CW Yang, XJ \\ Liu, L Zhao; (VI) Manuscript writing: All authors; (VII) Final approval of manuscript: All authors. \\ \#These authors contributed equally to this work. \\ Correspondence to: Professor Min Wu. Huaxi MR Research Center (HMRRC), Department of Radiology, West China Hospital, Sichuan University, \\ Chengdu, China. Email: wuminscu@scu.edu.cn; Professor Bin Song. Department of Radiology, West China Hospital, Sichuan University, Chengdu, \\ China. Email: songlab_radiology@163.com.
}

Background: To determine whether preoperative computed tomography (CT) features can be used for the prediction of gastrointestinal stromal tumors (GISTs) with a high Ki-67 proliferation index (Ki-67 PI).

Methods: A total of 198 patients with surgically and pathologically proven GISTs were retrospectively included. All GISTs were divided into a low Ki-67 PI group ( $<10 \%)$ and a high Ki-67 PI group ( $\geq 10 \%)$. All imaging features were blindly interpreted by two radiologists. Receiver operating characteristic (ROC) curve analyses were conducted to evaluate the predictive performance of the imaging features.

Results: Imaging features were found to be significantly different between the low and the high Ki-67 PI groups $(\mathrm{P}<0.05)$. Wall thickness of necrosis showed the highest predictive ability, with an area under the curve (AUC) of 0.838 [95\% confidence interval (CI): 0.627-0.957], followed by necrosis, necrosis degree, hyperenhancement of the overlying mucosa (HYOM), and long diameter (LD) (AUC >0.7, P<0.05). HYOM was the strongest predictive feature for the high Ki-67 PI GISTs group, with an odds ratio (OR) value of 30.037 (95\% CI: 5.707-158.106).

Conclusions: Imaging features, including the presence of necrosis, high necrosis degree, thick wall of necrosis, and HYOM were significant predictive indicators for the high Ki-67 PI GISTs group.

Keywords: Gastrointestinal stromal tumors (GIST); Ki-67 proliferation index (Ki-67 PI); computed tomography (CT); imaging features

Submitted Aug 20, 2021. Accepted for publication Oct 13, 2021.

doi: $10.21037 / \mathrm{atm}-21-4669$

View this article at: https://dx.doi.org/10.21037/atm-21-4669

\footnotetext{
^ ORCID: Cai-Wei Yang, 0000-0003-3335-3948; Xi-Jiao Liu, 0000-0002-6900-0696; Lian Zhao, 0000-0002-1304-9685; Feng Che, 00000002-8008-2696; Yuan Yin, 0000-0003-3359-4282; Hui-Jiao Chen, 0000-0002-6941-0089; Bo Zhang, 0000-0002-0254-5843; Min Wu, 0000-0002-7733-2498; Bin Song, 0000-0002-7269-2101.
} 


\section{Introduction}

Gastrointestinal stromal tumors (GISTs) are the most common mesenchymal tumors, originating from interstitial Cajal cells in the abdominal cavity or retroperitoneum (1). Immunohistochemical tests have demonstrated that GISTs are usually positive for CD117 and DOG1 proteins (2). The formation of GISTs is mainly due to mutations in the genes encoding the tyrosine kinase receptor KIT and platelet-derived growth factor receptor alpha (PDGFRA), resulting in the corresponding tyrosine kinase receptors becoming proto-oncogene drivers $(3,4)$. Some GISTs may develop distant metastases, with the most frequent sites of metastases being the peritoneum and the liver (5). It would be useful to assess the biological behaviors of GISTs before and after surgery, in order to make decisions regarding adjuvant therapy and treatment regimens for individual patients (5).

The cell proliferation index, also known as the Ki-67 proliferation index (Ki-67 PI), is the percentage of Ki-67positive staining cells in each cell population. Ki-67 PI is a crucial immunohistochemical marker for evaluating tumor heterogeneity and cell growth (6). The Ki-67 nucleoprotein is highly expressed in proliferating cells during the G1, S, and G2 phases based on mitotic count, and is downregulated in the G0 phase, implying involvement in cell proliferation, invasive aggressiveness, and malignant potential (6).

According to previous studies, a high level of Ki-67 PI is an independent predictive indicator for high-malignancy GISTs and poor survival prognosis (7-12). A high Ki-67 PI GIST indicated a reduced survival time and a poorer therapeutic response with molecular targeted treatment (13-17). Moreover, Ki-67 PI is significantly correlated with KIT or PDGFRA mutations of GISTs, which may assist individual multidisciplinary planning of gene-targeted therapy $(18,19)$. Therefore, preoperative non-invasive prediction of Ki-67 PI in GISTs would be very valuable. A previous study demonstrated that the Ki-67 PI is correlated with risk stratification and prognosis prediction of GISTs, and that computed tomography (CT) features are valuable in preoperative evaluation (20). However, the diagnostic performance and predictability of CT features is unknown and uncertain when determining high Ki-67 PI patients.

Therefore, our study was designed to explore the potential predictive ability of CT features. The purpose of this study is to determine whether enhanced CT features can be used for the preoperative prediction of
GISTs with high Ki-67 PI. Our study initially assesses the diagnostic performance and predictive ability of detailed CT quantitative and morphological imaging features in determining high Ki-67 PI GISTs.

We present the following article in accordance with the STARD reporting checklist (available at https://dx.doi. org/10.21037/atm-21-4669).

\section{Methods}

\section{Patients}

This retrospective study was approved by the institutional review board at West China Hospital, Sichuan University (No. 2020-249). All procedures performed in this study involving human participants were in accordance with the Declaration of Helsinki (as revised in 2013). Because of the retrospective nature of the research, the requirement for informed consent was waived. Our institutional pathological databases were searched using the following terms: "GISTs or gastrointestinal stromal tumors", from September 2010 to June 2019. A total of 794 patients were initially included. The inclusion criteria were as follows: (I) surgical specimens of GISTs were pathologically analyzed; (II) patients without any history of preoperative targeted therapy; (III) patients with only one single primary tumor (note: this study only evaluated the primary lesion for tumors with metastases); and (IV) cases where preoperative contrast-enhanced CT images were available, and the interval between the CT acquisition time and surgical time of GISTs was within 2 weeks. The exclusion criteria were as follows: (I) patients who were unavailable or had incomplete enhanced CT images in our hospital; and (II) GISTs without Ki-67 PI identifications in immunochemistry. Finally, 198 GISTs were included in this study (the study flowchart is displayed in Figure 1).

\section{CT scanning parameters}

Bowel cleansing was a precondition for CT examination, which involved a low-residue diet or ample fluids the day before the examination and fasting 10 hours prior to the examination. The patients were requested to drink $600-1,000 \mathrm{~mL}$ of water within $40-60 \mathrm{~min}$ before the examination.

All patients were examined using Brilliance 64 (Philips Medical System, Eindhoven, the Netherlands), 128-slice 


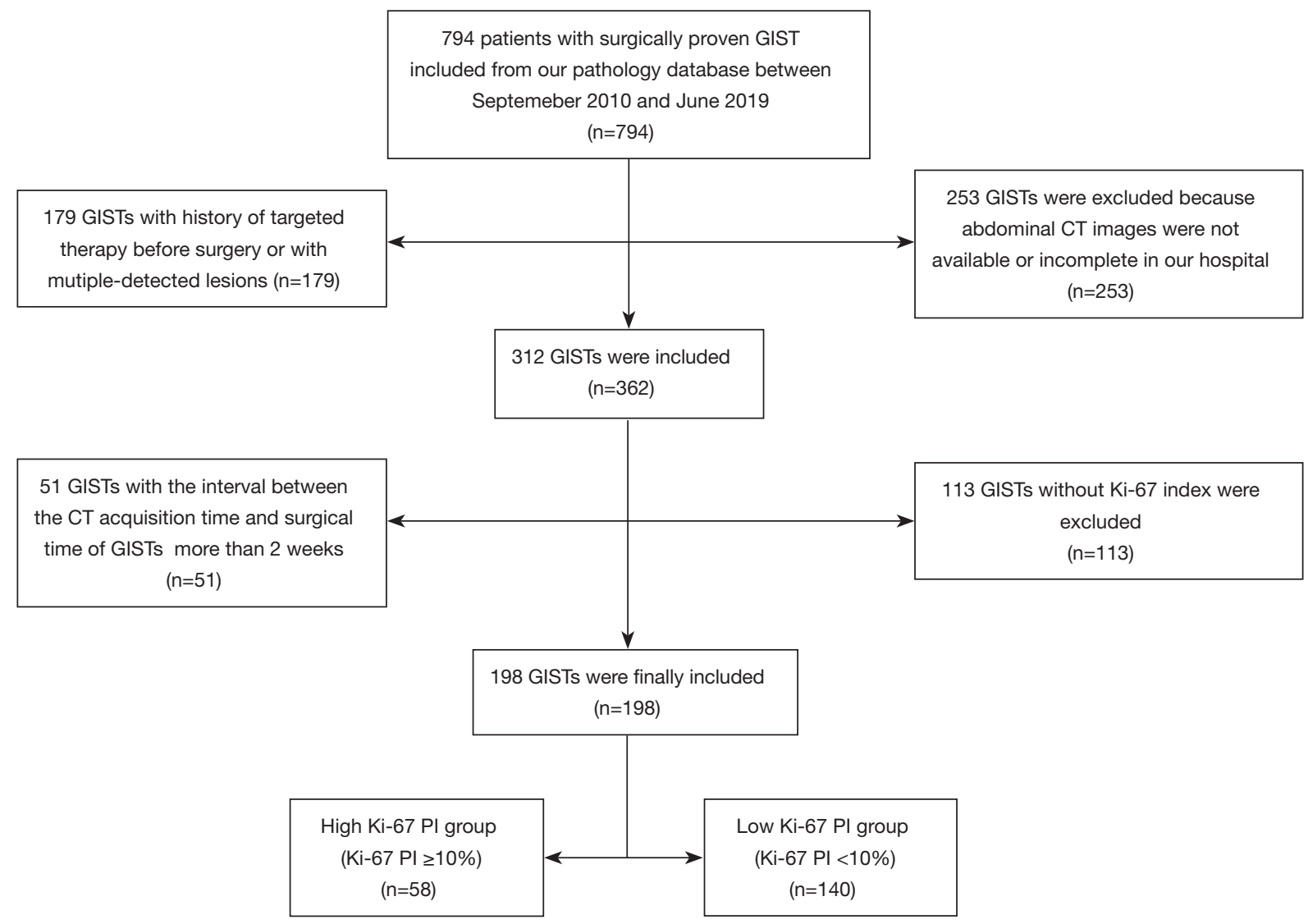

Figure 1 The flowchart illustrates the patient inclusion and exclusion process for the study cohort. GISTs, gastrointestinal stromal tumors; Ki-67 PI, Ki-67 proliferation index.

scanner (SOMATOM Definition AS+, Siemens Healthcare), or dual-source CT system (SOMATOM Definition Flash, Siemens Healthcare). The scanning range covered the entire abdomen. The scanning parameters were as follows: tube voltage, $120 \mathrm{kV}$; tube current, 145-200 mAs; slice thickness, $2-5 \mathrm{~mm}$; slice interval, $2 \mathrm{~mm}$; field of view, $35-50 \mathrm{~cm}$; matrix, $512 \times 512$; rotation time, $0.5 \mathrm{~s}$; and pitch 1.0. With the trigger threshold of the aorta reaching $170 \mathrm{HU}$, enhanced images were obtained with the arterial phase at the trigger, and the portal vein phase at $30 \mathrm{~s}$ after the trigger. Iodinated contrast agent $(1.2-1.5 \mathrm{~mL} / \mathrm{kg}$, Iopamiro, Bracco, Italy; Ultravist, Bayer, Germany) was injected intravenously at a flow rate of $2.5-3.0 \mathrm{~mL} / \mathrm{s}$ using a high-pressure syringe (Medrad Stellant CT Injector System, Medrad Inc.).

\section{CT image analysis}

All imaging feature assessments were independently interpreted by two radiologists (Lian Zhao and Xi-Jiao Liu, with 4 and 15 years of diagnostic experience in abdominal imaging, respectively) on Syngo Imaging Workplaces (VersionVB35A, Siemens AG, Erlangen, Germany). Precise quantitative imaging data were also recorded and calculated. The two radiologists were blinded to the clinical data and pathological results. Inconsistencies between the observers in the initial evaluation were settled by additional discussion to make a final determination.

The following qualitative CT features of the lesions were recorded: (I) locations, which were recorded as stomach, duodenum, intestine, rectum, or extra-gastrointestinal tract (including omentum, mesentery, and peritoneum); (II) borders, which were classified as ill-defined or welldefined; (III) contours, which were classified as round, ovoid [egg shaped with long diameter (LD)/short diameter (SD) ratio $\leq 1.5]$, dumbbell (shaped like two larger parts joined by a thinner neck), bell (shaped like an inverted cup and with a wider base), flat (growing in a certain axis 
and with LD/SD ratio >1.5), or irregular (no specific shape); (IV) growth types, which were classified into endoluminal, exophytic, or mixed (including intraluminal and extraluminal sections of the gastrointestinal tract contour) (21); (V) enhancement patterns, which were evaluated as homogeneous or heterogeneous; (VI) enhancement degrees, which were classified as mild, intermediate, or marked on the basis of tumor CT attenuation difference between the arterial and unenhanced phases [differences $\leq 20$ Hounsfield unit (HU) were defined as mild; 20-40 HU were defined as intermediate; and $\geq 40 \mathrm{HU}$ were defined as marked] (22); (VII) tumor enhancement levels, which were graded as slight, moderate, or serious, compared to the paraspinal muscle and the liver at the same level in the arterial phase (if the CT value of the tumor was less than the muscle, it was defined as slight; if it was between that of the muscle and the liver, it was defined as moderate; and if it was greater than that of the liver, it was defined as severe); (VIII) calcification; (IX) air density in mass (intraluminal air density connected to the gastrointestinal lumen); (X) ulceration (a focal discontinuity or break on the endoluminal surface of the tumor); (XI) enlarged vasculature feeding or draining the mass (EVFDM) (detectably enlarged or engorged veins or arteries near the tumor) (23); (XII) necrosis (an area with CT attenuation $\leq 20 \mathrm{HU}$ in each phase and enhancement difference of this area $\leq 10 \mathrm{HU}$ between the unenhanced and enhanced phases (24); (XIII) cystic degeneration (a thin-walled region within the tumor with $\mathrm{CT}$ attenuation ranging from 0 to $10 \mathrm{HU}$ and without enhancement in the venous phase); (XIV) hemorrhage; (XV) hyperenhancement of the overlying mucosa (HYOM, a hyper-enhanced mucosal line delineating the tumor) (25); (XVI) adjacent organ invasion (an unclear boundary of the tumor with blurred outline relative to adjacent structures); (XVII) lymphadenopathy, which was defined as an enlarged lymph node $\mathrm{SD} \geq 1 \mathrm{~cm}$; (XVIII) metastasis; (IXX) peritonitis; (XX) ascites; and (XXI) rupture, which was defined as a focal tissue defect on the extraluminal region of the tumor surface with secondary peritonitis, peritoneal effusion, peritoneal abscess, or hemoperitoneum (26). Identification disputes of rupture were finally decided by pathological determination.

The quantitative parameters of these tumors were also measured. The LD and SD of each lesion were recorded, and the LD/SD ratio was then calculated. For CT attenuationrelated measurements, a circular region of interest (ROI) in the tumor (approximate size $15-18 \mathrm{~mm}^{2}$ ) was applied in
HU. For the enhancement degree, CT attenuation of each tumor and the aorta at the same layer in each phase was recorded, while the enhancement level of the paraspinal muscle and liver at the same level in each phase was measured. To avoid tumor heterogeneity, tumor attenuation in three phases was measured by three circular ROIs placed on different parts of the tumor, avoiding adjacent tumor vessels, cystic degeneration, necrosis, air density, calcification, and contiguous gastrointestinal tract wall. The average of the three ROIs was then recorded as tumor attenuation in the plain phase (Tp), tumor attenuation in the arterial phase (Ta), and tumor attenuation in the venous phase (Tv). To normalize the individual circulation level of each patient with different injection rates and doses, the CT values of $\mathrm{Tp}$, Ta, and $\mathrm{Tr}$ were divided by the aorta CT values at the same layer and phase (Ap, Aa, and Av), which were recorded as standard tumor attenuation in the plain phase (Sp, Tp/Ap), standard tumor attenuation in the arterial phase ( $\mathrm{Sa}, \mathrm{Ta} / \mathrm{Aa})$, and standard tumor attenuation in the venous phase $(\mathrm{Sv}, \mathrm{Tv} / \mathrm{Av})$, respectively. For tumors with EVFDM, the diameter of the tumor feeding artery or drainage vein was carefully observed in the enhanced phase. For tumors with necrosis, the necrosis degree was the estimated ratio of the necrotic component to parenchymal part of the tumor using percentage count. Similarly, the wall thickness of necrosis was measured by the thickest distance between the solid tumor wall and necrotic component of the tumor in the enhanced phase.

\section{Histological evaluation and immunobistochemical assessment}

Surgically resected sections for histology and immunohistochemistry were matched to the significant parenchyma of GISTs as much as possible, where the calcification, and air density of the tumor was carefully avoided. Next, the predominant cell type (including spindle cells, epithelioid cells, or mixed spindle cells and epithelioid component) and mitotic count were recorded. All mitotic cells counted in 50 high-power fields (HPF) were recorded as $<5 / 50,5-10 / 50$, or $\geq 10 / 50 \mathrm{HPF}$. Ki-67 PI expression was assessed by immunohistochemistry within 7 days after surgery. The pathologist was blinded to clinical data and imaging assessments. Monoclonal rabbit antihuman Ki-67 antibody (Rabbit monoclonal, SP6, Abcam Shanghai) was used to detect the Ki-67 PI. GISTs were categorized into two groups: a high Ki-67 PI group (Ki-67 PI $\geq 10 \%$ ) and 
a low Ki-67 PI group (Ki-67 PI $<10 \%$ ) based on previous research (7-9,27). Risk stratification of modified National Institute of Health (mNIH) classification was eventually determined using histological evaluation (mitotic count) and clinical records (tumor site, tumor size, and presence of rupture).

\section{Statistical analysis}

Categorical data were calculated using Pearson's chisquare test or Fisher's exact test, and quantitative data were calculated using the Student's $t$-test or Mann-WhitneyWilcoxon test. Continuous data were analyzed using the normality test and presented as means \pm standard deviations, while categorical data were presented as numbers with percentages. Spearman's correlation analysis with correlation coefficient rho (r) was conducted to assess the strength of connection between significant qualitative or quantitative parameters and Ki-67 PI groups. Diagnostic performance was evaluated by the receiver operating characteristic (ROC) analysis, and the area under the ROC curve (AUC) was calculated. The comparison of different AUCs was performed using DeLong's test (28). The Youden index decided the corresponding sensitivity and specificity at the optimal cut-off value.

The interobserver agreement for qualitative features was evaluated using the kappa test. The intraclass correlation coefficient (ICC) was used to assess the interobserver and intra-observer agreement of the quantitative parameters (29). In multivariate logistic regression analysis, variables with a $\mathrm{P}$ value less than 0.05 determined by univariate analysis were selected as an independent indicator, and the odds ratio (OR) as the relative risk estimates with $95 \%$ confidence interval (CI) of each risk factor was obtained.

Statistical tests were performed using the Statistical Package for Social Sciences software version 25.0 (IBM, Chicago, IL, USA) and MedCalc statistical software (version 15.8; https://www.medcalc.org). A $\mathrm{P}$ value $<0.05$ (twotailed) was considered as statistically significant for each statistical test.

\section{Results}

\section{Patient demographics and pathological results}

In total, 198 GISTs patients (89 men and 109 women; average age: $56.83 \pm 12.46$ years) were included, with a median CT acquisition and surgical time interval of 9 days (range, 1-14 days). The detailed patient characteristics are presented in Table 1. Clinical symptoms included digestive tract hemorrhage ( $\mathrm{n}=76,38.4 \%$ ), abdominal discomfort or pain $(n=83,41.9 \%)$, and asymptomatic patients $(n=39$, $19.7 \%)$. All gastric GISTs patients $(n=140)$ underwent different surgical resections, including partial gastrectomy $(\mathrm{n}=96,68.6 \%)$, wedge resection $(\mathrm{n}=28,20.0 \%)$, and total gastrectomy ( $\mathrm{n}=16,11.4 \%)$.

A majority of the GISTs were spindle-cell tumors (156, $78.8 \%$ ), while epithelioid type and mixed type GISTs were seen in $17(8.6 \%)$ and $15(7.6 \%)$ patients, respectively. Mitotic counts ranged from 0 to 110 per 50 HPF (mean, 6.2 mitoses per $50 \mathrm{HPF}$ ). There were $\leq 5$ per $50 \mathrm{HPF}$ in 124 (62.6\%) patients, $5-10$ per $50 \mathrm{HPF}$ in $41(20.7 \%)$ patients, $\geq 10$ per $50 \mathrm{HPF}$ in $33(16.7 \%)$ patients. In terms of $\mathrm{mNIH}$ risk stratification, very low-risk, low-risk, intermediate-risk, and high-risk classifications were counted as 14 (7.1\%), 70 (35.4\%), 50 (25.3\%), 64 (32.3\%), respectively. Based on immunohistochemical assessment, 58 (29.3\%) tumors were identified as high Ki-67 PI (Figure 2), while 140 (70.7\%) were classified as the low Ki-67 PI group (Table 2).

There were no significant differences in gender, age, clinical symptom, and type of gastric surgery between the two groups of GIST patients $(\mathrm{P}>0.05)$.

\section{The correlation between Ki-67 PI GIST groups and clinical indexes}

The correlation between Ki-67 PI GIST groups and mNIH risk stratification or mitotic count is shown in Table 2. The incidence of intermediate- and high-risk stratification in the high Ki-67 PI group was markedly higher than that in the low $\mathrm{Ki}-67 \mathrm{PI}$ group, with a percentage ratio of 1.93 (82.79\% vs. $47.14 \%)$. Risk stratification and mitotic count were significantly different between the two groups $(\mathrm{P}<0.001)$. The Ki-67 PI GIST groups were positively correlated with risk stratification and mitotic count, and the rank correlation coefficients (r) were 0.425 and 0.524 , respectively.

\section{Imaging findings}

Of the qualitative CT features, an ill-defined border, heterogeneous enhancement pattern, air density in mass, ulceration, EVFDM, necrosis (Figures 3,4), cystic degeneration (Figure 4), hemorrhage, HYOM (Figure 4), 
Table 1 Demographic and clinical characteristics of included patients

\begin{tabular}{lccc}
\hline Characteristics & Total GISTs $(\mathrm{n}=198)$ & $\begin{array}{c}\text { High Ki-67 PI GISTs } \\
\text { group }(\mathrm{n}=58)\end{array}$ & $\begin{array}{c}\text { Low Ki-67 PI GISTs } \\
\text { group }(\mathrm{n}=140)\end{array}$ \\
\hline Age (mean \pm SD) & $56.83 \pm 12.46$ & $58.57 \pm 13.35$ & $56.11 \pm 12.04$ \\
Gender (male/female) & $89 / 109$ & $32 / 26$ & $57 / 83$ \\
Male (mean \pm SD) & $57.61 \pm 12.31$ & $58.69 \pm 13.95$ & $57.00 \pm 11.37$ \\
Female (mean \pm SD) & $56.20 \pm 12.60$ & $58.42 \pm 12.85$ & $55.51 \pm 12.51$ \\
Clinical symptom (n/\%) & & & 0.538 \\
Digestive tract hemorrhage & $76 / 38.4$ & $23 / 39.7$ & $53 / 37.9$ \\
Abdominal discomfort or pain & $83 / 41.9$ & $24 / 41.3$ & $59 / 42.1$ \\
Asymptomatic & $39 / 19.7$ & $11 / 19.0$ & $28 / 20.0$ \\
Type of gastric surgery ( $\mathrm{n}=140, \mathrm{n} / \%)$ & & & 0.969 \\
Partial gastrectomy & & $29 / 67.4$ & $67 / 69.1$ \\
Wedge resection & $96 / 68.6$ & $10 / 23.3$ & $18 / 18.5$ \\
Total gastrectomy & $28 / 20.0$ & $4 / 9.3$ & $12 / 12.4$ \\
\hline
\end{tabular}

*, between the high and low Ki-67 PI GISTs groups, categorical data were calculated using Pearson's chi-square or Fisher's exact tests, and quantitative data were calculated with the Student $t$-test or Mann-Whitney-Wilcoxon test. Ki-67 PI, Ki-67 proliferation index; GISTs, gastrointestinal stromal tumors.
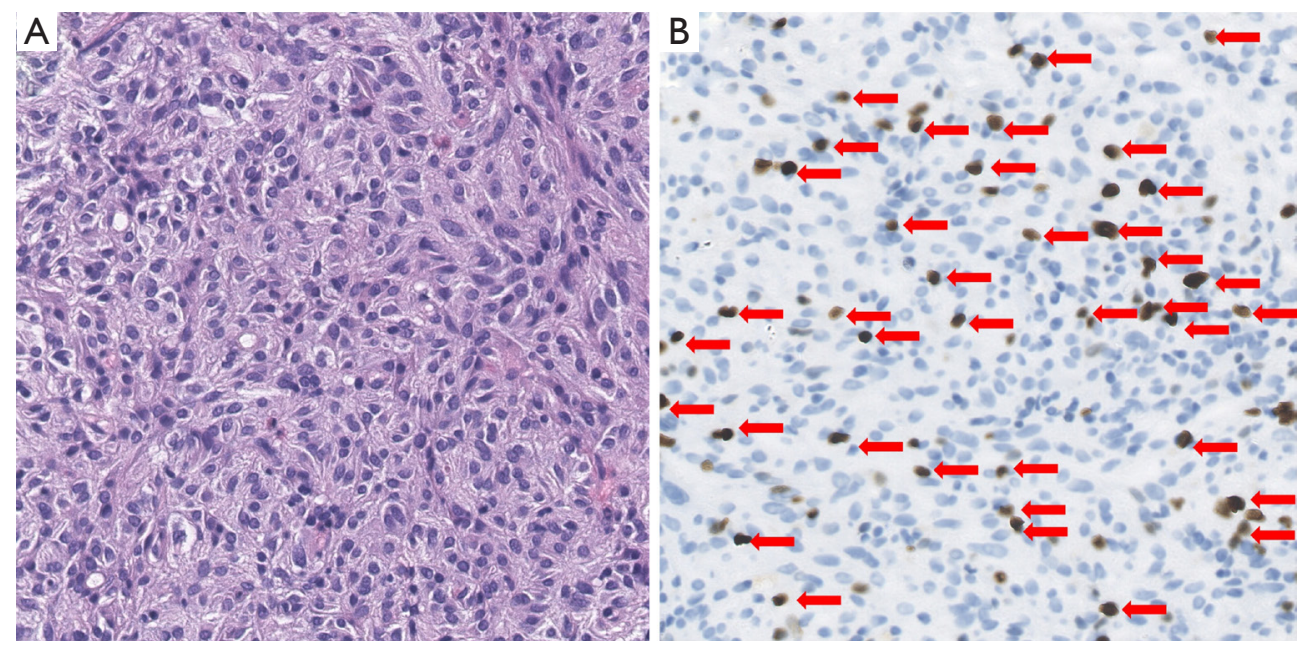

Figure 2 A gastrointestinal stromal tumor patient with high Ki-67 PI. (A) Hematoxylin and eosin image (H\&E staining, $\times 40)$ and (B) Ki-67 nuclear staining image (Ki-67 staining; magnification, $\times 40$ ). The analytic result of Ki-67 PI quantitation was $>10 \%$ with Ki-67-positive cells in red arrows (B). Ki-67 PI, Ki-67 proliferation index.

adjacent organ invasion, lymphadenopathy, metastasis, peritonitis, ascites, and rupture were found to be significantly different between the low and high Ki-67 PI groups $(\mathrm{P}<0.05$, Table 3). As for the quantitative CT features, $\mathrm{LD}, \mathrm{SD}, \mathrm{LD} / \mathrm{SD}$ ratio, $\mathrm{Tp}$, necrosis degree, wall thickness of necrosis, and diameter of EVFDM were found to be significantly different between the two groups $(\mathrm{P}<0.05$, Table 4). The Ta and Tv were not associated with low or 
Table 2 Correlation between Ki-67 PI GIST groups and mNIH risk stratification or mitotic count

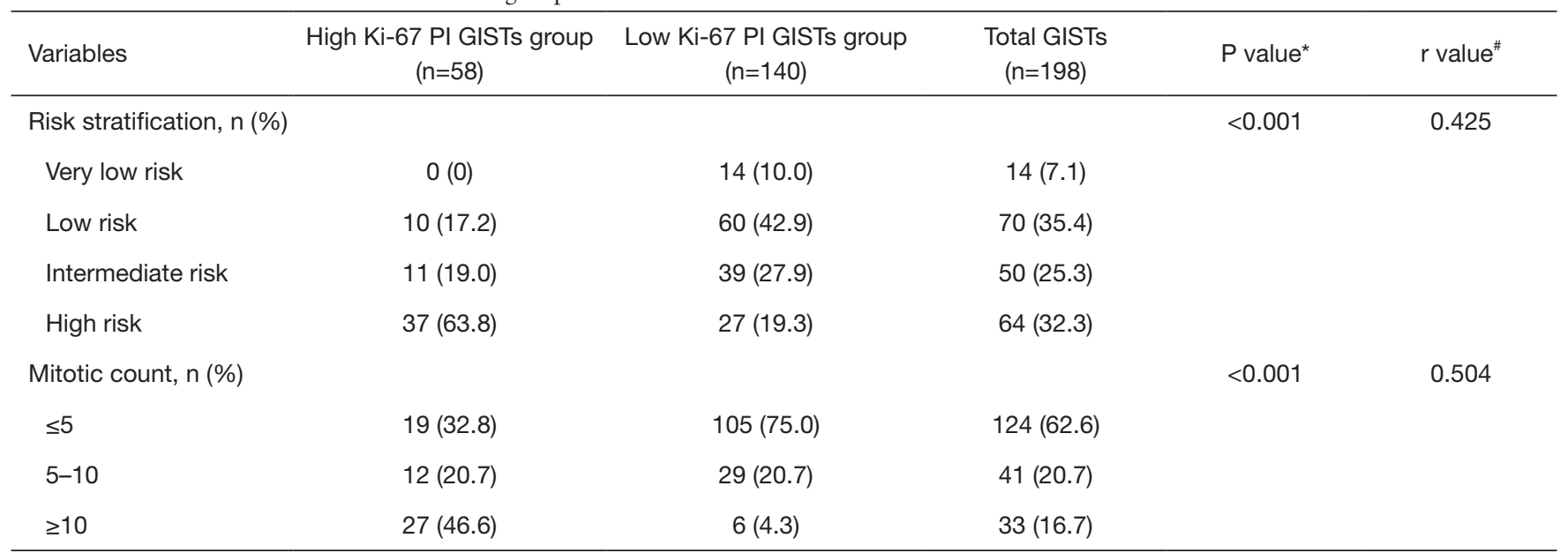

*, between the high and low Ki-67 PI GISTs groups, variables were calculated using Pearson's chi-square or Fisher's exact tests. \#, between the high and low Ki-67 PI GISTs groups, meaningful variables were analyzed with correlation analyses. Data are numbers of positive patients (with percentage of positive/total numbers in parentheses). Percentages may not add up to $100 \%$ because of rounding. Ki-67 PI, Ki-67 proliferation index; GISTs, gastrointestinal stromal tumors; mNIH, modified National Institute of Health.
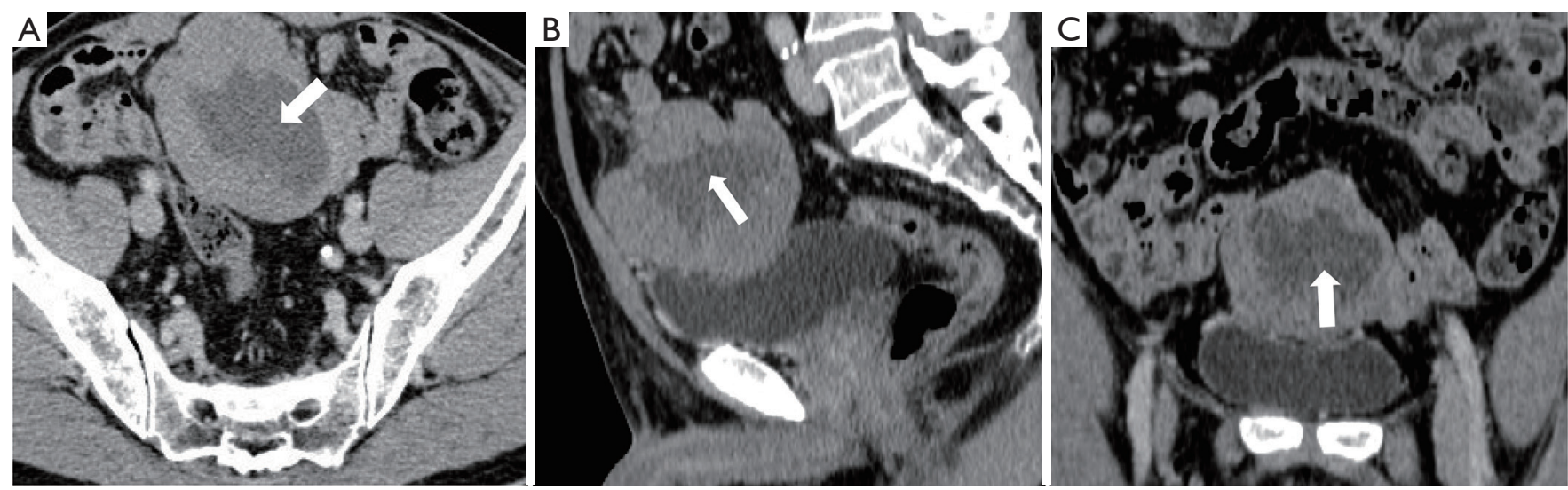

Figure 3 A high Ki-67 PI-group GIST in the small intestine of a 75-year-old male. Venous phase contrast-enhanced CT scan shows a welldefined, irregular border with heterogeneous enhancement and mixed growth pattern. Axial image (A), sagittal image (B), and coronal image (C) depict the presence of necrosis (white arrows). Ki-67 PI, Ki-67 proliferation index; GIST, gastrointestinal stromal tumor.

high Ki-67 PI classifications $(\mathrm{P}>0.05)$. The standard tumor attenuation in the unenhanced and enhanced phases (Sp, Sa, $\mathrm{Sv})$ showed no statistical differences between the two groups $(\mathrm{P}>0.05)$. These meaningful qualitative and quantitative features were correlated with the Ki-67 PI groups positively, except for $\mathrm{Tp}(\mathrm{r}=-0.181)$. As shown in Table 3 and Table 4, the rank correlation coefficient $(\mathrm{r})$ values ranged from 0.164 to 0.625 .

ROC curve analyses results showed that the wall thickness of necrosis, LD, HYOM, necrosis, and necrosis degree had good AUC values (AUC $>0.7, \mathrm{P}<0.05$, Table 5). Of these, wall thickness of necrosis demonstrated the highest predictive value, with an AUC of 0.838 (95\% CI: $0.627-0.957$ ), sensitivity of $76.9 \%$ (95\% CI: $46.2-$ $95.0 \%$ ), and specificity of $80.0 \%$ (95\% CI: 44.4-97.5\%), followed by necrosis, necrosis degree, HYOM, and LD with AUCs of 0.802 (95\% CI: 0.740-0.855), 0.795 (95\% CI: 0.732-0.849), 0.773 (95\% CI: 0.709-0.830), and 0.705 (95\% CI: 0.637-0.768), respectively (Figure 5). DeLong's test demonstrated that the AUCs did not differ significantly 

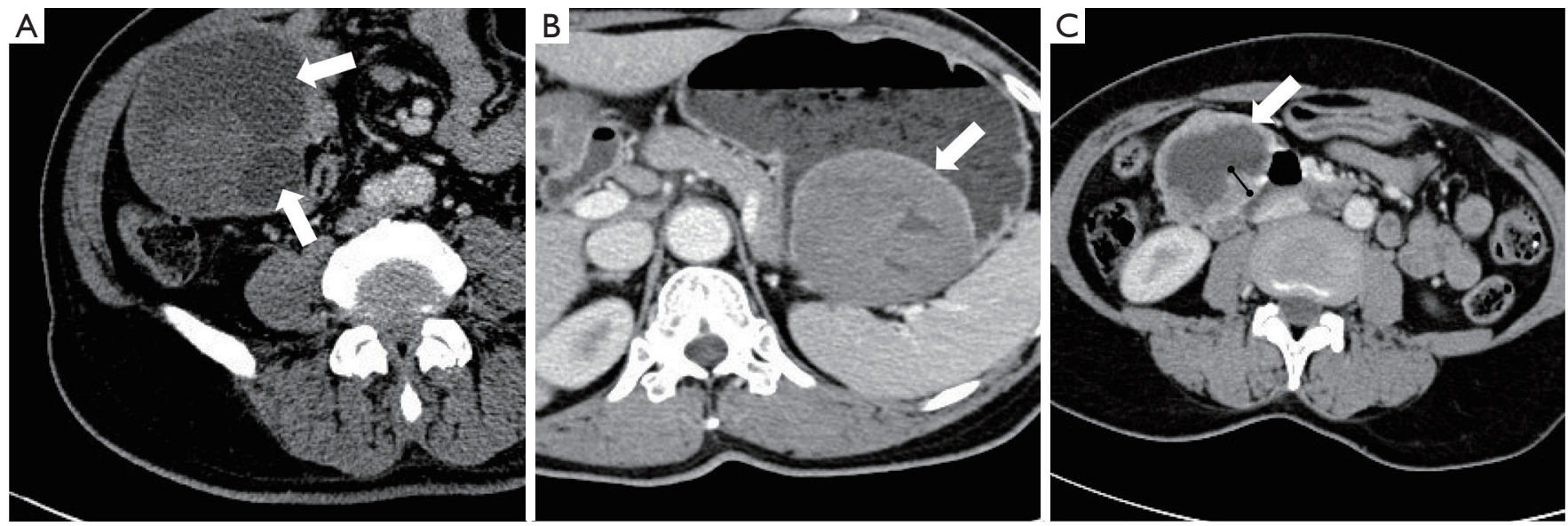

Figure 4 Various CT features of GISTs. (A) A high Ki-67 PI-group GIST in the small intestine of a 71-year-old male. Axial venous phase CT scan shows a well-defined, oval mass with heterogeneous enhancement, exophytic growth pattern, and multiple cystic degeneration in tumor (white arrow). (B) A high Ki-67 PI-group GIST in the fundus of stomach of a 62-year-old female. Axial venous phase CT scan shows a well-defined, bell-shaped mass with heterogeneous enhancement, endoluminal growth pattern, and hyperenhancement of the overlying mucosa (white arrow). (C) A high Ki-67 PI-group GIST in the small intestine of a 55-year-old female. Axial venous phase CT scan shows a well-defined, oval mass with heterogeneous enhancement, exophytic growth pattern, and depicts the presence of necrosis (white arrow). The black string measures the wall thickness of necrosis. Ki-67 PI, Ki-67 proliferation index; GIST, gastrointestinal stromal tumor.

between different features. Multinomial logistic regression analyses showed that the presence of necrosis $(\mathrm{OR}=2.987$, 95\% CI: $1.328-6.718)$, cystic degeneration $(\mathrm{OR}=14.057$, 95\% CI: $1.016-194.527)$, HYOM (OR $=30.037$, 95\% CI: $5.707-158.106)$, and $\mathrm{Tp} \leq 32 \mathrm{HU}(\mathrm{OR}=4.650,95 \%$ CI: $1.378-15.695)$ were determined to be independent predictors for the high Ki-67 PI GISTs group $(\mathrm{P}<0.05$, Table 6).

Both intra- and inter-observer ICCs for CT features were good, with kappa values of qualitative parameters ranging from 0.659 to 0.970 and ICC values of quantitative parameters ranging from 0.842 to $0.991(\mathrm{P}<0.05$, Table 3 and Table 4).

\section{Discussion}

The clinical signs of our study were consistent with the results of previous studies: the low and high Ki-67 PI GIST groups were not statistically different in terms of gender, age, clinical symptoms, and type of gastric surgery. The most common age range for GIST development was $55-60$ years, with a tendency to occur in women $(1,30,31)$. The most common clinical symptoms included abdominal discomfort or pain, digestive tract hemorrhage, and asymptomatic (in that order) $(1,32)$. Also, for gastric stromal tumors, patients underwent partial gastrectomy, followed by wedge resection, and total gastrectomy (in that order).

Our univariate analyses results showed that high Ki-67 PI GISTs tended to have an ill-defined border, heterogeneous enhancement pattern, and more frequently presented with air density in mass, ulceration, EVFDM, necrosis, cystic degeneration, hemorrhage, HYOM, adjacent organ invasion, lymphadenopathy, metastasis, peritonitis, ascites, and rupture, which was similar to previous findings (33-36). Moreover, $\mathrm{LD}, \mathrm{SD}, \mathrm{LD} / \mathrm{SD}$ ratio, $\mathrm{Tp}$, necrosis degree, wall thickness of necrosis, and diameter of EVFDM were found to be significantly different between the low and high Ki-67 PI groups. Furthermore, our study demonstrated that the Ki67 PI groups were correlated to mitotic count and $\mathrm{mNIH}$ risk classification, and these CT features were correlated to Ki-67 PI groups. In the present study, the differences of Sp, Sa, Sv, Ta, and Tv between the two groups were not significant. The same is true for the relationship between $\mathrm{CT}$ attenuation in the three phases and mNIH risk stratification of GISTs $(24,37)$. The relationship between CT attenuation and Ki-67 PI levels of GISTs remains unclear and controversial.

ROC curve analyses demonstrated that wall thickness of necrosis, necrosis, necrosis degree, HYOM, and LD achieved good AUC values (AUC >0.7). However, through 
Table 3 Qualitative CT features between the high and low Ki-67 PI GISTs groups

\begin{tabular}{|c|c|c|c|c|c|c|}
\hline Variables & $\begin{array}{c}\text { Total GISTs }(n=198), \\
n(\%)\end{array}$ & $\begin{array}{l}\text { High Ki-67 PI GISTs } \\
\text { group }(n=58), n(\%)\end{array}$ & $\begin{array}{l}\text { Low Ki-67 PI GISTs } \\
\text { group }(n=140) \text {, n (\%) }\end{array}$ & $P$ value ${ }^{\star 1}$ & $r$ value ${ }^{* 2}$ & $\begin{array}{l}\text { Kappa } \\
\text { value }^{\star 3}\end{array}$ \\
\hline Location & & & & 0.261 & - & 0.946 \\
\hline Stomach & $140(70.7)$ & $43(74.1)$ & 97 (69.3) & & & \\
\hline Duodenum & $21(10.6)$ & $3(5.2)$ & 18 (12.9) & & & \\
\hline Extra-gastrointestinal tract ${ }^{{ }^{4}}$ & $7(3.5)$ & $4(6.9)$ & $3(2.1)$ & & & \\
\hline Borders & & & & 0.021 & 0.164 & 0.906 \\
\hline III-defined & $62(31.3)$ & $25(43.1)$ & $37(26.4)$ & & & \\
\hline Ovoid & $101(51.0)$ & $27(46.6)$ & $74(52.9)$ & & & \\
\hline Dumbbell & $50(25.3)$ & 19 (32.8) & $31(22.1)$ & & & \\
\hline Bell & $7(3.5)$ & $1(1.7)$ & $6(4.3)$ & & & \\
\hline Flat & $1(0.5)$ & $1(1.7)$ & 0 & & & \\
\hline Irregular & $15(7.6)$ & $5(8.6)$ & $10(7.1)$ & & & \\
\hline Growth types & & & & 0.059 & - & 0.904 \\
\hline Endoluminal & $53(26.8)$ & $11(19.0)$ & $42(30.0)$ & & & \\
\hline Enhancement degrees & & & & 0.857 & - & 0.970 \\
\hline Mild & $82(41.4)$ & $23(39.7)$ & $59(42.1)$ & & & \\
\hline Intermediate & $56(28.3)$ & $18(32.1)$ & $38(27.1)$ & & & \\
\hline Marked & $60(30.3)$ & $17(28.3)$ & $43(30.7)$ & & & \\
\hline Enhancement levels & & & & 0.963 & - & 0.954 \\
\hline Slight & $66(33.3)$ & $20(34.5)$ & $46(32.9)$ & & & \\
\hline Moderate & $68(34.3)$ & $20(34.5)$ & 48 (34.3) & & & \\
\hline Severe & 64 (32.3) & $18(31.0)$ & 46 (32.9) & & & \\
\hline
\end{tabular}

Table 3 (continued) 
Table 3 (continued)

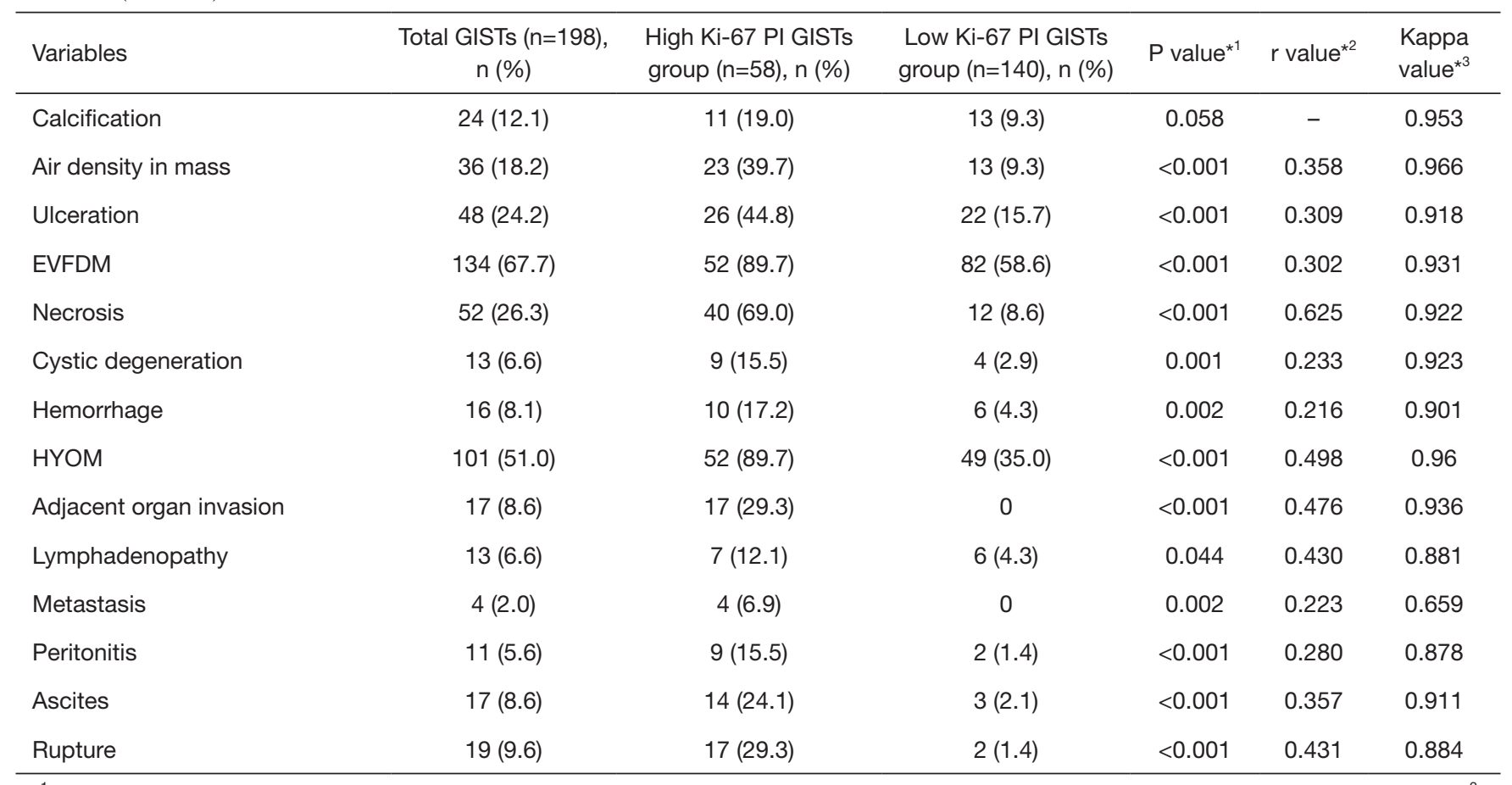

*1, between the high and low Ki-67 PI GISTs groups, variables were calculated using Pearson's chi-square or Fisher's exact tests; *2, between the high and low Ki-67 PI GISTs groups, meaningful variables were analyzed using correlation analyses; ${ }^{*}$, the inter-observer agreement for qualitative features was evaluated using the kappa test; ${ }^{*}$, extra-gastrointestinal tract included omentum, mesentery, and peritoneum. Data are numbers of positive patients (with percentage of positive/total numbers in parentheses). Percentages may not add up to $100 \%$ because of rounding. Ki-67 PI, Ki-67 proliferation index; GISTs, gastrointestinal stromal tumors; EVFDM, enlarged vasculature feeding or draining the mass; HYOM, hyperenhancement of the overlying mucosa.

multinominal logistic regression analyses, only necrosis, cystic degeneration, HYOM, and Tp $\leq 32 \mathrm{HU}$ were determined to be independent predictors for the high Ki67 PI GISTs group. Larger tumor LD could be a high-risk factor for GISTs based on the mNIH stratification (38). This is consistent with our study, where larger LD tended to occur in high Ki-67 PI GISTs. In terms of HYOM, which might be caused by mucosal disruption, invasion, or ulceration, patients presenting with HYOM tend to have high-risk stratification of gastric GISTs (25). Even if necrosis and its related parameters were not significant indicators for ruptured GISTs according to previous studies (26), they were demonstrated to be significant predictors in high-risk stratification GISTs $(24,33,39)$. Previous studies have also shown that necrosis is one of the pathological predictors of malignant behavior of GISTs and is more important for evaluating the prognosis of GISTs $(17,36,40-42)$. It is considered that necrosis is directly related to the severe proliferation of tumor portion; that is, necrosis will occur in the most invasive and aggressive area (42).

Ki-67 PI was previously used to predict the malignancy of GISTs $(8,13,15,43,44)$, and was found to have prognostic value in GISTs (16). Zhao et al. reported that Ki-67 PI classifications $(\leq 5 \%, 5-8 \%$, and $>8 \%)$ independently predict recurrence-free survival of GISTs, which could complement $\mathrm{mNIH}$ classification to differentiate various prognoses accurately and effectively in high-risk GIST patients. High-risk (Ki-67 PI >8\%) GISTs showed poorer therapeutic responses with imatinib treatment (13). As a useful complement to $\mathrm{mNIH}$ classification, the preoperative prediction of high Ki-67 PI levels can provide additional and individual data for clinical treatment decision-making. Recent studies have explored the value of Ki-67 PI levels in the prognostic assessment of GISTs. A study concluded that high Ki-67 PI GISTs ( $\geq 10 \%)$ indicated reduced overall survival and disease-free survival (17). Belev et al. found that a high Ki-67 PI $(\geq 6 \%)$ is statistically significant in terms 
Table 4 Quantitative CT features between the high and low Ki-67 PI GISTs groups

\begin{tabular}{|c|c|c|c|c|c|c|}
\hline Variables & $\begin{array}{l}\text { Total GISTs }(n=198) \\
\quad(\text { mean } \pm \text { SD })\end{array}$ & $\begin{array}{l}\text { High Ki-67 PI GISTs group } \\
\quad(n=58),(\text { mean } \pm \text { SD })\end{array}$ & $\begin{array}{l}\text { Low Ki-67 PI GISTs group } \\
\quad(n=140),(\text { mean } \pm \text { SD) }\end{array}$ & $P$ value ${ }^{\star 1}$ & $r$ value ${ }^{\star 2}$ & ICC value ${ }^{* 3}$ \\
\hline LD (mm) & $53.56 \pm 34.79$ & $68.31 \pm 37.87$ & $47.45 \pm 31.61$ & $<0.001$ & 0.274 & 0.991 \\
\hline LD/SD & $1.28 \pm 0.31$ & $1.35 \pm 0.47$ & $1.24 \pm 0.20$ & 0.028 & 0.157 & 0.922 \\
\hline Tv (HU) & $81.68 \pm 30.85$ & $77.03 \pm 26.97$ & $83.60 \pm 32.32$ & 0.174 & & 0.969 \\
\hline $\mathrm{Sp}(\mathrm{HU})$ & $0.87 \pm 0.23$ & $0.85 \pm 0.25$ & $0.88 \pm 0.22$ & 0.523 & & 0.913 \\
\hline $\mathrm{Sa}(\mathrm{HU})$ & $0.27 \pm 0.12$ & $0.25 \pm 0.12$ & $0.28 \pm 0.13$ & 0.16 & & 0.884 \\
\hline $\begin{array}{l}\text { Diameter of EVFDM } \\
(\mathrm{mm})\end{array}$ & $2.20 \pm 1.91$ & $2.93 \pm 1.71$ & $1.89 \pm 1.92$ & $<0.001$ & 0.248 & 0.855 \\
\hline
\end{tabular}

${ }^{* 1}$, between the high and Ki-67 PI GISTs groups, quantitative variables were calculated using the Student's $t$-test or Mann-WhitneyWilcoxon test; ${ }^{* 2}$, between the high and low Ki-67 PI GISTs groups, meaningful quantitative variables were analyzed using correlation analyses; ${ }^{*}$, the ICC was used to assess the inter-observer and intra-observer agreement of quantitative parameters. Ki-67 PI, Ki-67 proliferation index; GISTs, gastrointestinal stromal tumors; LD, long diameter; SD, short diameter; Tp, tumor attenuation in plain phase; Ta, tumor attenuation in arterial phase; Tv, tumor attenuation in venous phase; Sp, standard tumor attenuation in plain phase; Sa, standard tumor attenuation in arterial phase; Sv, standard tumor attenuation in venous phase; EVFDM, enlarged vasculature feeding or draining the mass; ICC, intraclass correlation coefficient.

of recurrence and identified that Ki-67 PI is an important prognostic predictor for recurrence and that is crucial for assessing the malignant potential of GISTs (14).

Another previous study investigated the correlation between Ki-67 PI, CT features, and risk stratification in GISTs, and explored the prognosis of CT features and the Ki-67 PI in GISTs (20). The results showed that the occurrence of GISTs of high $\mathrm{mNIH}$ risk classification or metastasis and the mitotic count were significantly higher in the $\mathrm{Ki}-67 \mathrm{PI}>5 \%$ groups compared to the $\mathrm{Ki}$ $67 \mathrm{PI} \leq 5 \%$ group $(\mathrm{P}<0.001)$, with the Ki-67 PI found to be positively correlated with $\mathrm{mNIH}$ risk stratification $(\mathrm{r}=0.558)$ or mitotic count $(\mathrm{r}=0.619)$ (20). Moreover, the significance of preoperative Ki-67 PI and mNIH risk stratification evaluation was effectively confirmed by CT features, including tumor sizes, contours, borders, necrosis, cystic degeneration, and enhancement patterns (20). Thus, the correlation between CT features and Ki-67 PI was demonstrated to make decisions regarding follow-up care and disease management for GISTs before surgery. However, this study did not assess the diagnostic performance and predictive ability of CT features in determining high Ki-67 PI group. Our results indicated that necrosis, necrosis degree, wall thickness of necrosis, $\mathrm{LD}$, and HYOM could effectively diagnoses high Ki67 PI GISTs from low Ki-67 PI GISTs preoperatively. Furthermore, necrosis, cystic degeneration, HYOM, and $\mathrm{Tp} \leq 32 \mathrm{HU}$ could also reliably predict the high Ki-67 PI GISTs group.

There have been some studies investigated on conventional imaging features and radiomics to predict the gene mutations of GISTs, but there is no study explored between Ki-67 PI and KIT or PDGFRA mutations in GISTs. Our study did not perform the correlation between Ki-67 PI and gene mutations in GISTs, because we did not have the gene mutation results from the histological data. If we have a chance, we will continue to conduct further research. 
Table 5 Predictive performance of CT features

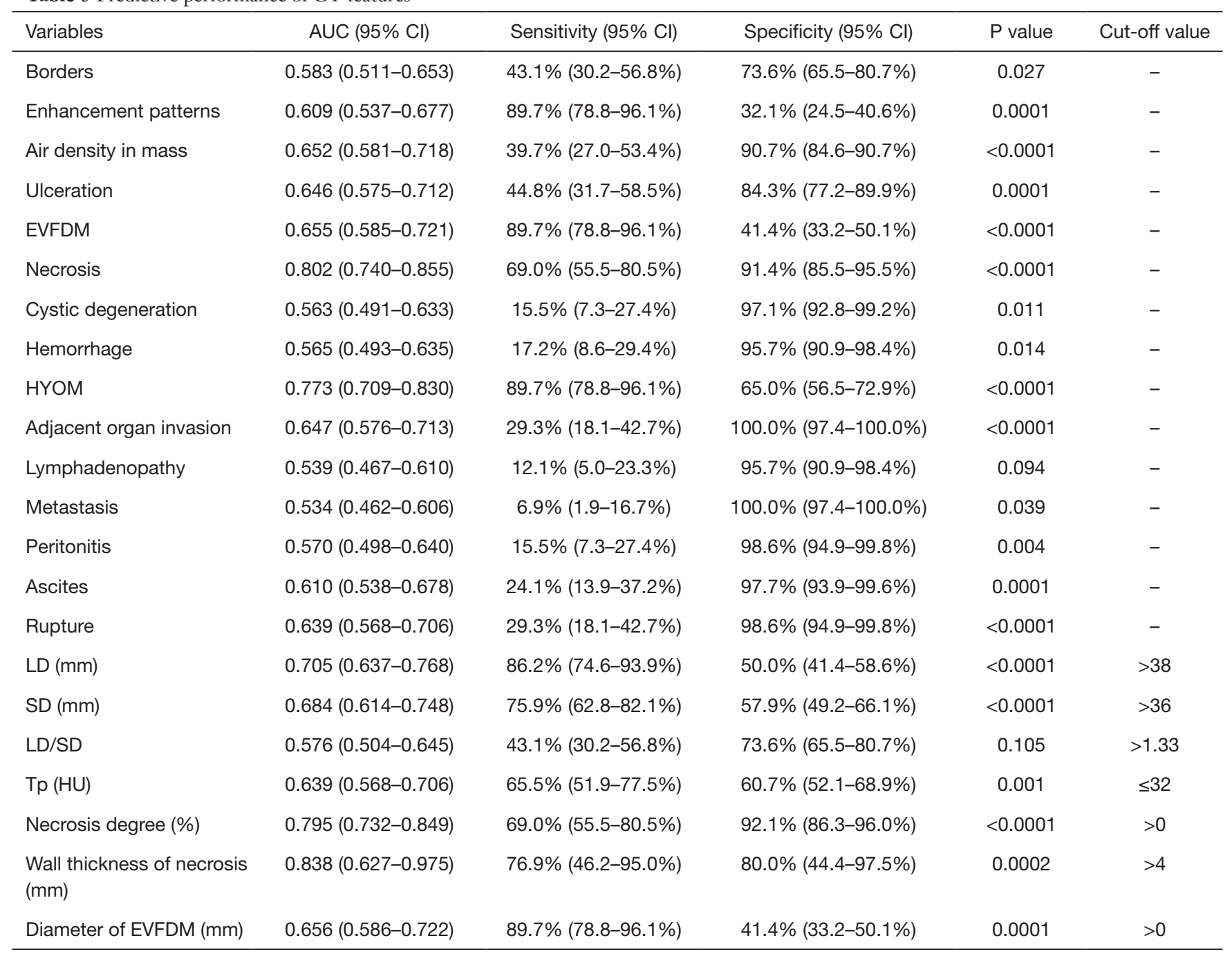

AUC, area under the curve; Cl, confidential interval; EVFDM, enlarged vasculature feeding or draining the mass; HYOM, hyperenhancement of the overlying mucosa; LD, long diameter; SD, short diameter; Tp, tumor attenuation in plain phase.

Our study has some limitations that should be noted. Firstly, this was a retrospective study. Secondly, we only included GISTs patients with surgical resection and immunohistochemical assessment of Ki-67 PI, which may have caused selection bias. Finally, due to the lack of follow-up and prognosis-related data, we did not perform prognostic analyses.

In conclusion, CT imaging features, including necrosis, high necrosis degree, thick wall of necrosis, and HYOM were significant predictive indicators for the high Ki67 PI GISTs group. Also, necrosis, cystic degeneration, HYOM, and $\mathrm{Tp} \leq 32 \mathrm{HU}$ could be independent predictive factors for high Ki-67 PI GISTs. Preoperative enhanced CT imaging features may help to predict the high Ki67 PI GISTs and provide more information for clinicians. Contrast-enhanced CT could be a noninvasive substitute of GISTs for histological evaluation and immunohistochemical assessment in clinical practice. 
ROC curves of significant CT features

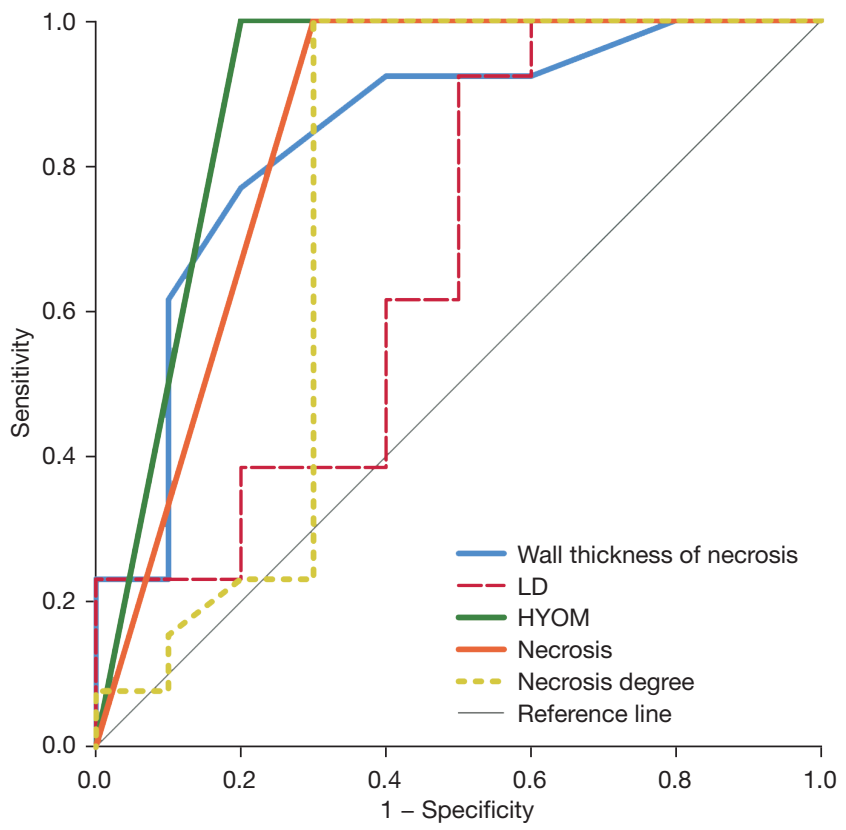

Figure 5 ROC curves of wall thickness of necrosis, LD, HYOM, necrosis, and necrosis degree for predicting the high Ki-67 PI group. The AUCs are 0.838 (95\% CI: 0.627-0.957) for wall thickness of necrosis, 0.705 (95\% CI: 0.637-0.768) for LD, 0.773 (95\% CI: 0.709-0.830) for HYOM, 0.802 (95\% CI: 0.740-0.855) for necrosis, and 0.795 (95\% CI: 0.732-0.849) for necrosis degree. ROC, receiver operating characteristic; AUC, area under the curve; HYOM, hyperenhancement of the overlying mucosa; LD, long diameter; Ki-67 PI, Ki-67 proliferation index.

Table 6 Multinomial logistic regression analyses for predicting high Ki-67 PI GISTs

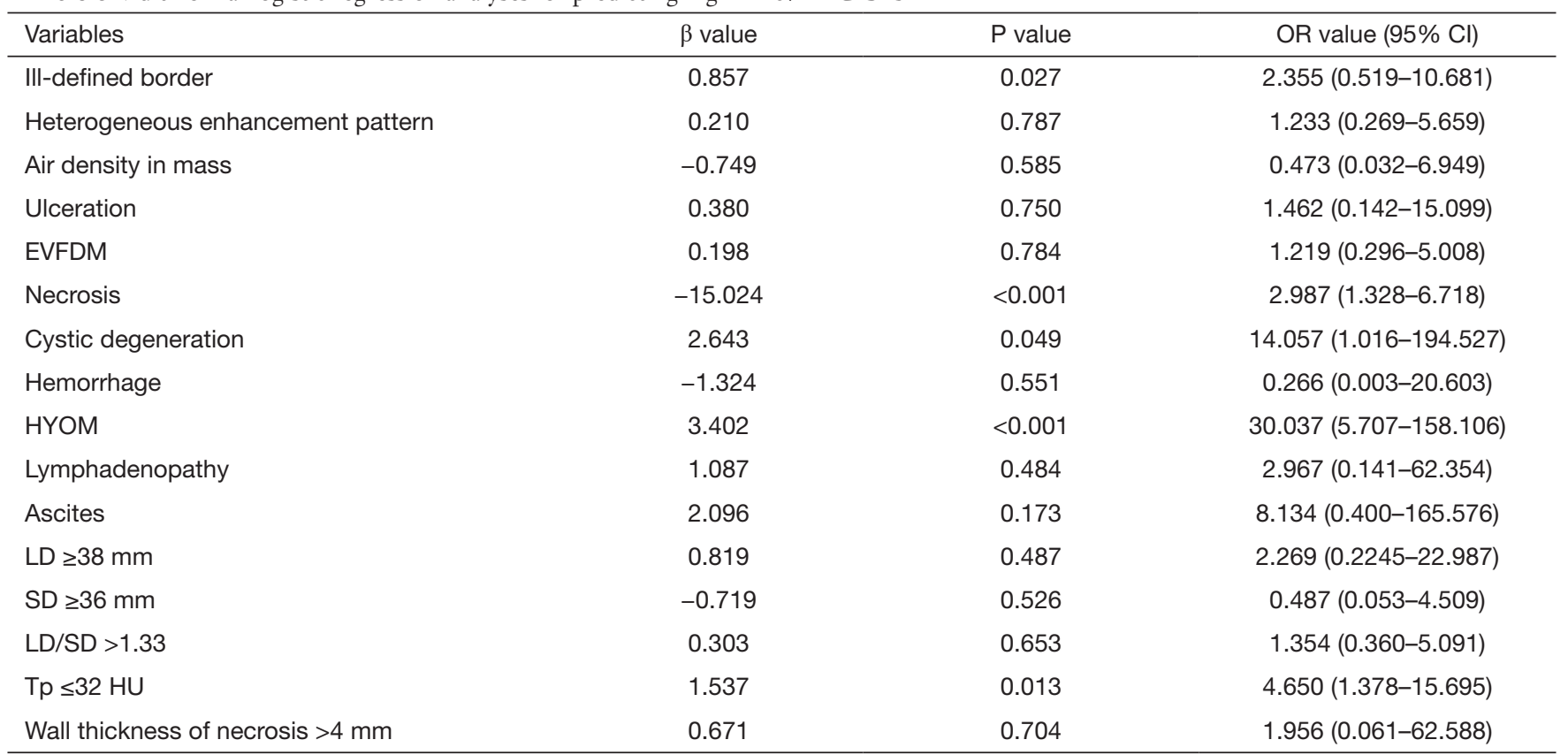

Ki-67 PI, Ki-67 proliferation index; GISTs, gastrointestinal stromal tumors; EVFDM, enlarged vasculature feeding or draining the mass; HYOM, hyperenhancement of the overlying mucosa; LD, long diameter; SD, short diameter; Tp tumor attenuation in plain phase. 


\section{Acknowledgments}

Funding: This work was supported by the National Nature Science Foundations of China (grant number: 82001810) and the 1.3.5 project for disciplines of the ExcellenceClinical Research Incubation Project, West China Hospital, Sichuan University (grant number: 19HXFH054).

\section{Footnote}

Reporting Checklist: The authors have completed the STARD reporting checklist. Available at https://dx.doi. org/10.21037/atm-21-4669

Data Sharing Statement: Available at https://dx.doi. org/10.21037/atm-21-4669

Conflicts of Interest: All authors have completed the ICMJE uniform disclosure form (available at https://dx.doi. org/10.21037/atm-21-4669). Dr. BS serves as an unpaid Associate Editor-in-chief of Annals of Translational Medicine from Sept 2020 to Aug 2022. The other authors have no conflicts of interest to declare.

Ethical Statement: The authors are accountable for all aspects of the work in ensuring that questions related to the accuracy or integrity of any part of the work are appropriately investigated and resolved. This retrospective study was approved by the institutional review board at West China Hospital, Sichuan University (No. 2020-249). All procedures performed in this study involving human participants were in accordance with the Declaration of Helsinki (as revised in 2013). Because of the retrospective nature of the research, the requirement for informed consent was waived.

Open Access Statement: This is an Open Access article distributed in accordance with the Creative Commons Attribution-NonCommercial-NoDerivs 4.0 International License (CC BY-NC-ND 4.0), which permits the noncommercial replication and distribution of the article with the strict proviso that no changes or edits are made and the original work is properly cited (including links to both the formal publication through the relevant DOI and the license). See: https://creativecommons.org/licenses/by-nc-nd/4.0/.

\section{References}

1. Joensuu H, Hohenberger P, Corless CL. Gastrointestinal stromal tumour. Lancet 2013;382:973-83.

2. Hirota S, Isozaki K, Moriyama $\mathrm{Y}$, et al. Gain-of-function mutations of c-kit in human gastrointestinal stromal tumors. Science 1998;279:577-80.

3. Heinrich MC, Corless CL, Duensing A, et al. PDGFRA activating mutations in gastrointestinal stromal tumors. Science 2003;299:708-10.

4. Joensuu H, Rutkowski P, Nishida T, et al. KIT and PDGFRA mutations and the risk of GI stromal tumor recurrence. J Clin Oncol 2015;33:634-42.

5. Judson I, Bulusu R, Seddon B, et al. UK clinical practice guidelines for the management of gastrointestinal stromal tumours (GIST). Clin Sarcoma Res 2017;7:6.

6. Scholzen T, Gerdes J. The Ki-67 protein: from the known and the unknown. J Cell Physiol 2000;182:311-22.

7. Jiang J, Jin MS, Suo J, et al. Evaluation of malignancy using Ki-67, p53, EGFR and COX-2 expressions in gastrointestinal stromal tumors. World J Gastroenterol 2012;18:2569-75.

8. Wang X, Mori I, Tang W, et al. Helpful parameter for malignant potential of gastrointestinal stromal tumors (GIST). Jpn J Clin Oncol 2002;32:347-51.

9. Nanding A, Tang L, Cai L, et al. Low ING4 protein expression detected by paraffin-section immunohistochemistry is associated with poor prognosis in untreated patients with gastrointestinal stromal tumors. Gastric Cancer 2014;17:87-96.

10. Nakamura N, Yamamoto H, Yao T, et al. Prognostic significance of expressions of cell-cycle regulatory proteins in gastrointestinal stromal tumor and the relevance of the risk grade. Hum Pathol 2005;36:828-37.

11. Lu C, Liu L, Wu X, et al. CD133 and Ki-67 expression is associated with gastrointestinal stromal tumor prognosis. Oncol Lett 2013;6:1289-94.

12. Tsumuraya M, Kato H, Miyachi K, et al. Comprehensive analysis of genes involved in the malignancy of gastrointestinal stromal tumors. Anticancer Res 2010;30:2705-15.

13. Zhao WY, Xu J, Wang M, et al. Prognostic value of Ki67 index in gastrointestinal stromal tumors. Int J Clin Exp Pathol 2014;7:2298-304.

14. Belev B, Brčić I, Prejac J, et al. Role of Ki-67 as a prognostic factor in gastrointestinal stromal tumors. World J Gastroenterol 2013;19:523-7.

15. Aoyagi K, Kouhuji K, Yano S, et al. Malignant potential of gastrointestinal stromal tumor of the stomach. Int Surg 2009;94:1-9.

16. Artigiani Neto R, Logullo AF, Stávale JN, et al. Ki- 
67 expression score correlates to survival rate in gastrointestinal stromal tumors (GIST). Acta Cir Bras 2012;27:315-21.

17. Turkel Kucukmetin N, Cicek B, Saruc M, et al. Ki67 as a prognostic factor for long-term outcome following surgery in gastrointestinal stromal tumors. Eur J Gastroenterol Hepatol 2015;27:1276-80.

18. Haller F, Cortis J, Helfrich J, et al. Epithelioid/mixed phenotype in gastrointestinal stromal tumors with KIT mutation from the stomach is associated with accelerated passage of late phases of the cell cycle and shorter diseasefree survival. Mod Pathol 2011;24:248-55.

19. Barreca A, Fornari A, Bonello L, et al. KIT and PDGFRA mutations and PDGFRA immunostaining in gastrointestinal stromal tumors. Mol Med Rep 2011;4:3-8.

20. Li H, Ren G, Cai R, et al. A correlation research of Ki67 index, CT features, and risk stratification in gastrointestinal stromal tumor. Cancer Med 2018;7:4467-74.

21. Kim HC, Lee JM, Kim KW, et al. Gastrointestinal stromal tumors of the stomach: CT findings and prediction of malignancy. AJR Am J Roentgenol 2004;183:893-8.

22. Janowitz P, Meier F, Reisig J. Gastric schwannoma as a rare differential diagnosis of pleural effusion. Z Gastroenterol 2002;40:925-8.

23. Brancatelli G, Federle MP, Grazioli L, et al. Focal nodular hyperplasia: CT findings with emphasis on multiphasic helical CT in 78 patients. Radiology 2001;219:61-8.

24. Su Q, Wang Q, Zhang H, et al. Computed tomography findings of small bowel gastrointestinal stromal tumors with different histologic risks of progression. Abdom Radiol (NY) 2018;43:2651-8.

25. Peng G, Huang B, Yang X, et al. Preoperative CT feature of incomplete overlying enhancing mucosa as a highrisk predictor in gastrointestinal stromal tumors of the stomach. Eur Radiol 2021;31:3276-85.

26. Kim JS, Kim HJ, Park SH, et al. Computed tomography features and predictive findings of ruptured gastrointestinal stromal tumours. Eur Radiol 2017;27:2583-90.

27. Peker K, Sayar I, Gelincik I, et al. The diagnostic importance of matrix metalloproteinase-7 and nestin in gastrointestinal stromal tumors. Med Sci Monit 2014;20:674-80.

28. DeLong ER, DeLong DM, Clarke-Pearson DL. Comparing the areas under two or more correlated receiver operating characteristic curves: a nonparametric approach. Biometrics 1988;44:837-45.

29. Aerts HJ, Velazquez ER, Leijenaar RT, et al. Decoding tumour phenotype by noninvasive imaging using a quantitative radiomics approach. Nat Commun 2014;5:4006.

30. Zhang X, Ning L, Hu Y, et al. Prognostic Factors for Primary Localized Gastrointestinal Stromal Tumors After Radical Resection: Shandong Gastrointestinal Surgery Study Group, Study 1201. Ann Surg Oncol 2020;27:2812-21.

31. Miettinen M, Sobin LH, Lasota J. Gastrointestinal stromal tumors of the stomach: a clinicopathologic, immunohistochemical, and molecular genetic study of 1765 cases with long-term follow-up. Am J Surg Pathol 2005;29:52-68.

32. Mucciarini C, Rossi G, Bertolini F, et al. Incidence and clinicopathologic features of gastrointestinal stromal tumors. A population-based study. BMC Cancer 2007;7:230.

33. Zhou C, Duan X, Zhang X, et al. Predictive features of CT for risk stratifications in patients with primary gastrointestinal stromal tumour. Eur Radiol 2016;26:3086-93.

34. Tateishi U, Hasegawa T, Satake M, et al. Gastrointestinal stromal tumor. Correlation of computed tomography findings with tumor grade and mortality. J Comput Assist Tomogr 2003;27:792-8.

35. Choi IY, Yeom SK, Cha J, et al. Feasibility of using computed tomography texture analysis parameters as imaging biomarkers for predicting risk grade of gastrointestinal stromal tumors: comparison with visual inspection. Abdom Radiol (NY) 2019;44:2346-56.

36. Burkill GJ, Badran M, Al-Muderis O, et al. Malignant gastrointestinal stromal tumor: distribution, imaging features, and pattern of metastatic spread. Radiology 2003;226:527-32.

37. Wang JK. Predictive value and modeling analysis of MSCT signs in gastrointestinal stromal tumors (GISTs) to pathological risk degree. Eur Rev Med Pharmacol Sci 2017;21:999-1005.

38. Joensuu H. Risk stratification of patients diagnosed with gastrointestinal stromal tumor. Hum Pathol 2008;39:1411-9.

39. Vasconcelos RN, Dolan SG, Barlow JM, et al. Impact of CT enterography on the diagnosis of small bowel gastrointestinal stromal tumors. Abdom Radiol (NY) 2017;42:1365-73.

40. de Oliveira RP, Portari Filho PE, Iglesias AC, et al. Comparative study of the different degrees of risk of gastrointestinal stromal tumor. Rev Col Bras Cir 
Page 16 of 16

2015;42:32-6.

41. Tryggvason G, Gíslason HG, Magnússon MK, et al. Gastrointestinal stromal tumors in Iceland, 1990-2003: the icelandic GIST study, a population-based incidence and pathologic risk stratification study. Int J Cancer 2005;117:289-93.

42. Hou YY, Lu SH, Zhou Y, et al. Stage and histological grade of gastrointestinal stromal tumors based on a new approach are strongly associated with clinical behaviors. Mod Pathol 2009;22:556-69.

43. Lopes LF, Ojopi EB, Bacchi CE. Gastrointestinal stromal

Cite this article as: Yang CW, Liu XJ, Zhao L, Che F, Yin Y, Chen HJ, Zhang B, Wu M, Song B. Preoperative prediction of gastrointestinal stromal tumors with high $\mathrm{Ki}-67$ proliferation index based on CT features. Ann Transl Med 2021;9(20):1556. doi: $10.21037 /$ atm-21-4669

\section{Yang et al. Prediction of high Ki-67 index in GISTs with CT}

tumor in Brazil: clinicopathology, immunohistochemistry, and molecular genetics of 513 cases. Pathol Int 2008;58:344-52.

44. Gillespie V, Baer K, Farrelly J, et al. Canine gastrointestinal stromal tumors: immunohistochemical expression of CD34 and examination of prognostic indicators including proliferation markers Ki67 and AgNOR. Vet Pathol 2011;48:283-91.

(English Language Editor: A. Kassem) 